Homology, Homotopy and Applications, vol.18(2), 2016, pp.169-176

\title{
ON THE $f$-INVARIANT OF PRODUCTS
}

\section{HANNO VON BODECKER}

\author{
(communicated by Nicholas J. Kuhn)
}

\begin{abstract}
The $f$-invariant is a higher version of the $e$-invariant that takes values in the divided congruences between modular forms; in the situation of a cartesian product of two framed manifolds, the $f$-invariant can actually be computed from the $e$-invariants of the factors. The purpose of this note is to determine the $f$-invariant of all such products.
\end{abstract}

\section{Introduction and statement of the result}

In order to gain a better understanding of the stable homotopy groups of the sphere, which, by the Pontrjagin-Thom construction, can be interpreted as the bordism groups of framed manifolds, it proves helpful to organize the information using suitable invariants. In his seminal work on the $J$-homomorphism, Adams considered the $d$-invariant, which is essentially the degree of a map, and introduced the notion of an $e$-invariant $[\mathbf{1}]$. Using complex $K$-theory, he produced an invariant

$$
e_{\mathbb{C}}: \pi_{2 k+1}^{s t} \rightarrow \mathbb{Q} / \mathbb{Z},
$$

and determined its image; to be precise, $e_{\mathbb{C}}$ maps:

(i) $\pi_{8 k+1}^{s t}$ onto integer multiples of $\frac{1}{2}$,

(ii) $\pi_{8 k+3}^{s t}$ onto integer multiples of $\frac{B_{4 k+2}}{4 k+2}$,

(iii) $\pi_{8 k+5}^{s t}$ onto integer multiples of 1 ,

(iv) $\pi_{8 k+7}^{s t}$ onto integer multiples of $\frac{B_{4 k+4}}{8 k+8}$.

Moreover, he showed that the optimal values in (ii) and (iv) are attained on the generators of $\operatorname{Im} J$ in the corresponding dimensions (although $\operatorname{Im} J_{8 k+3}$ actually has twice the order of the denominator of $B_{4 k+2} /(4 k+2)$, which can be seen by making use of $K O$-theory), and constructed an 8-periodic family $\mu_{8 k+1}$ (in coker $J_{8 k+1}$ if $k>1$ ) detected by (i). The subsequent work of Conner and Floyd using bordism theory allowed the $e_{\mathbb{C}}$-invariant to be computed via the Todd genus of a complex null-bordism of the framed manifold in question [6]; even later, the work of Atiyah, Patodi, and Singer on index theory on manifolds with boundary [3] led to an analytic formulation of the $e$-invariant [4], which was used by Deninger and Singhof to

Received June 1, 2015, revised February 1, 2016; published on September 14, 2016.

2010 Mathematics Subject Classification: 55Q45.

Key words and phrases: stable homotopy group, $f$-invariant.

Article available at http://dx.doi.org/10.4310/HHA.2016.v18.n2.a8

Copyright (C) 2016, International Press. Permission to copy for private use granted. 
exhibit an infinite family of nilmanifolds representing (twice) the generator of $\operatorname{Im} J$ in dimensions $8 k+3(8 k+7)[\mathbf{7}]$.

Much of the recent progress on our knowledge of $\pi_{*}^{s t}$ can be accredited to the Adams-Novikov spectral sequence (ANSS),

$$
E_{2}^{p, q}=\operatorname{Ext}_{M U_{*} M U}^{p, q}\left(M U_{*}, M U_{*}\right) \Rightarrow \pi_{q-p}^{s t}
$$

and the rich algebraic structure inherent in complex oriented cohomology theories (see, e.g., [12]). Within this framework, the complex $e$-invariant can be considered as taking values in the 1-line of the ANSS, i.e.,

$$
e_{\mathbb{C}}: \pi_{2 k+1}^{s t} \rightarrow E_{2}^{1,2 k+2} \subseteq \mathbb{Q} / \mathbb{Z}
$$

Working locally at a prime $p$ and switching to $B P$, the 1-line is generated by the alpha elements $\alpha_{i / j} \in \operatorname{Ext}^{1,2(p-1) i}$ of order $p^{j}$; at odd primes, $j=1+\nu_{p}(i)$, and all these alphas are permanent and detect the generators of $\operatorname{Im} J_{(p)}$. At $p=2$ however, the situation is a little bit more subtle: The elements $\alpha_{4 k+3}$ are not permanent; furthermore $j=2+\nu_{2}(i)$ for $i=2 t>2$, but for $t=2 k+1$ it is actually $\alpha_{4 k+2 / 2}$ (i.e., twice the generator of $\mathrm{Ext}^{1,8 k+4}$ if $k>0$ ) that is a permanent cycle represented by an element in $\operatorname{Im} J$ of order eight (cf. [12, Theorem 5.3.7]).

In order to detect second filtration phenomena, Laures introduced the $f$-invariant, which is a follow-up to the $e$-invariant and takes values in the divided congruences between modular forms $[\mathbf{9}, \mathbf{1 0}]$. Let us briefly recall its definition: Considering the congruence subgroup $\Gamma=\Gamma_{1}(N)$, set $\mathbb{Z}^{\Gamma}=\mathbb{Z}\left[\zeta_{N}, 1 / N\right]$ and denote by $M_{*}^{\Gamma}$ the graded ring of modular forms w.r.t. $\Gamma$ which expand integrally, i.e., which lie in $\mathbb{Z}^{\Gamma} \llbracket q \rrbracket$. The ring of divided congruences $D^{\Gamma}$ consists of those rational combinations of modular forms which expand integrally; this ring can be filtered by setting

$$
D_{k}^{\Gamma}=\left\{f=\sum_{i=0}^{k} f_{i} \mid f_{i} \in M_{i}^{\Gamma} \otimes \mathbb{Q}, f \in \mathbb{Z}^{\Gamma} \llbracket q \rrbracket .\right.
$$

Finally, introduce

$$
\underline{\underline{D}}_{k}^{\Gamma}=D_{k}^{\Gamma}+M_{0}^{\Gamma} \otimes \mathbb{Q}+M_{k}^{\Gamma} \otimes \mathbb{Q} .
$$

Now, if $E l l^{\Gamma}$ denotes the complex oriented elliptic cohomology theory associated to the universal curve over the ring of modular forms w.r.t. $\Gamma$, the composite

$$
E_{2}^{2,2 k+2}[M U] \rightarrow E_{2}^{2,2 k+2}\left[E l l^{\Gamma}\right] \rightarrow \underline{\underline{D}}_{k+1}^{\Gamma} \otimes \mathbb{Q} / \mathbb{Z}
$$

is injective (away from primes dividing the level $N$ ) and can be combined with the composite $\pi_{2 k}^{s t} \rightarrow E_{\infty}^{2,2 k+2}[M U] \rightarrow E_{2}^{2,2 k+2}[M U]$ (for the second map, note that there are no differentials hitting the 2 -line). Then, for a fixed level $N$ (which we suppress from the notation), the $f$-invariant becomes a map

$$
f: \pi_{2 k}^{s t} \rightarrow \underline{\underline{D}}_{k+1}^{\Gamma} \otimes \mathbb{Q} / \mathbb{Z}
$$

In a more geometrical fashion, the $f$-invariant can be formulated as an elliptic genus of manifolds with corners of codimension two [10]; as shown in the author's thesis [13], this allows to use techniques from index theory to understand and, at least in some cases, to calculate the $f$-invariant analytically (see also [5] for a slightly different - analytical approach to the $f$-invariant). In the situation of the product of two framed manifolds, the $f$-invariant can actually be computed from the 
$e_{\mathbb{C}}$-invariants of the factors. The purpose of this note is to determine the $f$-invariant of all such products:

Theorem 1.1. Let $x_{4 k-1}$ be a generator of $\operatorname{Im} J$ in dimension $4 k-1$ and let $\mu_{8 k+1}$ be a representative of the $\mu$-family constructed by Adams. Then, for the level $N=3$, we have:
(i) $f\left(x_{3}^{2}\right) \equiv \frac{1}{2}\left(\frac{E_{1}^{2}-1}{12}\right)^{2}$,
(ii) $f\left(x_{7}^{2}\right) \equiv \frac{1}{2}\left(\frac{E_{4}-1}{240}\right)^{2}$,
(iii) $f\left(\mu_{8 k+1} \mu_{8 k^{\prime}+1}\right) \equiv \frac{1}{2} \frac{E_{1}-1}{2}$,
(iv) $f\left(\mu_{8 k+1} x_{8 k^{\prime}-1}\right) \equiv \frac{1}{2} \frac{E_{4}-1}{240}$,
(v) $f\left(\mu_{8 k+1} x_{8 k^{\prime}+3}\right) \equiv 0$.

Furthermore, for any level $N>1$, we have:

(vi) $f\left(x_{4 k-1} x_{4 k^{\prime}-1}\right) \equiv 0$,

unless $k=k^{\prime}$ equals one or two.

Some comments are in order before presenting the proof:

First of all, we have to admit that at even levels $N=2 l$, i.e., when two is inverted, our theorem does not provide anything new, since the products of alpha elements at odd primes are known to vanish [11] (although our result does not rely on this fact). At odd levels however, things become more interesting:

It might be somewhat amusing to find the two 8-periodic families (iii) and (iv), each (within its family) admitting the same representative of the $f$-invariant, but the experienced reader will surely recognize these as corresponding to $\mu_{8\left(k+k^{\prime}\right)+2}$ in coker $J$ and $\eta x_{8\left(k+k^{\prime}\right)-1}$ in $\operatorname{Im} J$, respectively.

Furthermore, our result shows that beyond these two families, only the exceptional cases (i) and (ii) (corresponding to the Kervaire elements of product type) admit a non-trivial $f$-invariant.

Summarizing, and bearing in mind that we work on the level of manifolds, i.e., representatives of permanent cycles, this theorem may be thought of as an elliptic (and strengthened) analog of [12, Theorem 5.5.8].

\section{2. $\quad$ Proof of Theorem 1.1}

The determination of the $f$-invariant of a product from the $e_{\mathbb{C}}$-invariants of its factors is made possible by the following result:

Lemma 2.1. [13] Let $Y_{1}, Y_{2}$ be odd-dimensional framed manifolds, and let $m\left(Y_{i}\right)$ be any modular form of weight $\left(\operatorname{dim} Y_{i}+1\right) / 2$ w.r.t. the fixed congruence subgroup $\Gamma=\Gamma_{1}(N)$ such that $\bar{m}\left(Y_{i}\right)=m\left(Y_{i}\right)-e_{\mathbb{C}}\left(Y_{i}\right) \in \mathbb{Z}^{\Gamma} \llbracket q \rrbracket$. Then we have

$$
f\left(Y_{1} \times Y_{2}\right) \equiv \bar{m}\left(Y_{1}\right) e_{\mathbb{C}}\left(Y_{2}\right) \equiv-\bar{m}\left(Y_{2}\right) e_{\mathbb{C}}\left(Y_{1}\right) .
$$

In particular, the $f$-invariant of a product is antisymmetric under exchange of the factors.

Besides the knowledge of the possible values of the $e_{\mathbb{C}}$-invariant, we need some elementary number theory: 
Lemma 2.2. For $n \geqslant 1$ and any positive integer $d$, we have:

(i) $\left(d^{p^{n-1}(p-1)}-1\right) d^{n} \equiv 0 \bmod p^{n}$,

(ii) $\left(d^{\left(2 n^{\prime}+1\right) 2^{n}}-1\right) d^{n+2} \equiv 0 \bmod 2^{n+2}$.

Proof. Part (i) is a simple consequence of the Euler-Fermat theorem (cf., e.g., [2]) which states that for $(a, m)=1$, we have:

$$
a^{\varphi(m)} \equiv 1 \bmod m,
$$

where $\varphi(m)=m \prod_{p \mid m}\left(1-\frac{1}{p}\right)$ is the Euler totient.

The refinement (ii) stems from the fact that for odd $d^{2 n^{\prime}+1}=2 k+1$ :

$$
\begin{aligned}
(2 k+1)^{2^{n}} & =1+2^{n} 2 k+\frac{2^{n}\left(2^{n}-1\right)}{2} 4 k^{2}+\sum_{i \geqslant 3}\left(\begin{array}{c}
2^{n} \\
i
\end{array}\right)(2 k)^{i} \\
& =1+2^{n+1}\left(k+\left(2^{n}-1\right) k^{2}\right)+\sum_{i \geqslant 3}\left(\begin{array}{c}
2^{n} \\
i
\end{array}\right)(2 k)^{i} \\
& \equiv 1 \quad \bmod 2^{n+2},
\end{aligned}
$$

since $\nu_{2}(i !)<i$ and $\nu_{2}\left(\left(2^{n}\right) ! /\left(2^{n}-i\right) !\right) \geqslant n+1$ for $i \geqslant 3$.

Concerning modular forms, recall that for even $k>2$ the Eisenstein series

$$
E_{k}=-\frac{2 k}{B_{k}} G_{k}=1-\frac{2 k}{B_{k}} \sum_{n=1}^{\infty} \sigma_{k-1}(n) q^{n}
$$

is a modular form of weight $k$ w.r.t. the full modular group. Furthermore recall (cf., e.g., [8, Section 3.2]) that the ring of modular forms w.r.t. $\Gamma=\Gamma_{1}(3)$ is generated by

$$
\begin{aligned}
& E_{1}=1+6 \sum_{n=1}^{\infty} \sum_{d \mid n}\left(\frac{d}{3}\right) q^{n}, \\
& E_{3}=1-9 \sum_{n=1}^{\infty} \sum_{d \mid n}\left(\frac{d}{3}\right) d^{2} q^{n},
\end{aligned}
$$

where $(\dot{)})$ is the Legendre Symbol; in passing, we note that $\left(E_{1}^{3}-E_{3}\right) / 27 \in \mathbb{Z} \llbracket q \rrbracket$. These modular forms satisfy the following useful congruence:

\section{Lemma 2.3.}

$$
\frac{1}{2}\left\{\frac{E_{1}^{2}-1}{12}+(2 k+1) \frac{E_{3}-1}{9}\right\} \in \mathbb{Z} \llbracket q \rrbracket
$$

Proof. Since

$$
E_{1}^{2}=1+12 \sum_{n=1}^{\infty} \sum_{3 \nmid d \mid n} d q^{n}
$$

we have to show that

$$
\frac{1}{2}\left\{\sum_{n=1}^{\infty} \sum_{3 \nmid d \mid n} d q^{n}-(2 k+1) \sum_{n=1}^{\infty} \sum_{d \mid n}\left(\frac{d}{3}\right) d^{2} q^{n}\right\} \in \mathbb{Z} \llbracket q \rrbracket,
$$


but obviously

$$
\sum_{d \mid n}\left(\frac{d}{3}\right) d^{2} \equiv \sum_{3 \nmid d \mid n} d^{2} \equiv \sum_{3 \nmid d \mid n} d \quad \bmod 2
$$

Now let us prove the Theorem:

\section{The exceptional cases (i) and (ii)}

Looking at the list, we may assume that $e_{\mathbb{C}}\left(x_{3}\right)$ is given by $-\frac{1}{12}$; of course, this is in accordance with the well-known fact that $x_{3}$ may be represented by the sphere $S^{3}$ (with the framing induced by quaternion multiplication). So we apply Lemma 2.1, choose

$$
-\bar{m}\left(x_{3}\right)=\frac{E_{1}^{2}-1}{12}=\sum_{n=1}^{\infty} \sum_{3 \nmid d \mid n} d q^{n} \in \mathbb{Z} \llbracket q \rrbracket,
$$

and compute

$$
\frac{1}{12} \frac{E_{1}^{2}-1}{12} \equiv-\frac{1}{2}\left(\frac{E_{1}^{2}-1}{12}\right)^{2} \equiv \frac{1}{2}\left(\frac{E_{1}^{2}-1}{12}\right)^{2} \bmod \underline{\underline{D}}_{4}^{\Gamma}
$$

this proves (i).

Similarly, $x_{7}$ may be represented by the sphere $S^{7}$ (see, e.g., [6]), resulting in $e_{\mathbb{C}}\left(x_{7}\right)=\frac{1}{240}$, so we choose

$$
\bar{m}\left(x_{7}\right)=\frac{E_{4}-1}{240}=\sum_{n=1}^{\infty} \sum_{d \mid n} d^{3} q^{n} \in \mathbb{Z} \llbracket q \rrbracket,
$$

and 'complete the square', hence establishing (ii).

Remark 2.4. Of course, (i) and (ii) correspond to the Kervaire elements of product type, i.e., $\alpha_{2 / 2}^{2}=\beta_{2 / 2}$ and $\alpha_{4 / 4}^{2}=\beta_{4 / 4}$ at the prime $p=2$. In order to see this on the level of $f$-invariants, we may compare (i) and (ii) to the results of [8], i.e.,

$$
\begin{gathered}
f\left(\beta_{2 / 2}\right) \equiv \frac{1}{2}\left(\frac{E_{1}^{2}-1}{4}\right)^{2} \in \underline{\underline{D}}_{4}^{\Gamma} \otimes \mathbb{Q} / \mathbb{Z}, \\
f\left(\beta_{4 / 4}\right) \equiv \frac{1}{2}\left(\frac{E_{1}^{2}-1}{4}\right)^{4}+\frac{1}{2}\left(\frac{E_{1}^{2}-1}{4}\right)^{3} \in \underline{\underline{D}}_{8}^{\Gamma} \otimes \mathbb{Q} / \mathbb{Z} .
\end{gathered}
$$

Concerning (ii), the fourth Eisenstein series is a modular form of weight four, hence it follows that $E_{4}=9 E_{1}^{4}-8 E_{1} E_{3}$ by comparing the first two terms of the $q$-expansions. 
Consequently, a short computation modulo $\underline{\underline{D}}_{8}^{\Gamma}$ reveals:

$$
\begin{aligned}
\frac{1}{2}\left(\frac{E_{4}-1}{16}\right)^{2} & =\frac{1}{2}\left(\frac{1}{2} \frac{E_{1}^{4}-1}{8}+\frac{1}{2}\left(E_{1}^{4}-E_{1} E_{3}\right)\right)^{2} \\
& =\frac{1}{2}\left(\frac{1}{4}\left(\frac{E_{1}^{4}-1}{8}\right)^{2}+\frac{1}{2} \frac{E_{1}^{4}-1}{8}\left(E_{1}^{4}-E_{1} E_{3}\right)+\frac{1}{4}\left(E_{1}^{4}-E_{1} E_{3}\right)^{2}\right) \\
& \equiv \frac{1}{32} E_{1} E_{3}+\frac{1}{2}\left(\frac{E_{1}^{2}-1}{4}\right)^{4}+\frac{1}{2}\left(\frac{E_{1}^{2}-1}{4}\right)^{3}+\frac{1}{8}\left(\frac{E_{1}^{2}-1}{4}\right)^{2} \\
& \equiv \frac{1}{16} \frac{E_{1}^{2}-1}{4}+\frac{1}{2}\left(\frac{E_{1}^{2}-1}{4}\right)^{4}+\frac{1}{2}\left(\frac{E_{1}^{2}-1}{4}\right)^{3}+\frac{1}{16} \frac{E_{1}^{4}-1}{8}-\frac{1}{16} \frac{E_{1}^{2}-1}{4} \\
& \equiv \frac{1}{2}\left(\frac{E_{1}^{2}-1}{4}\right)^{4}+\frac{1}{2}\left(\frac{E_{1}^{2}-1}{4}\right)^{3},
\end{aligned}
$$

since

$$
\begin{aligned}
-\frac{1}{32} E_{1} E_{3} & \equiv \frac{1}{2} \frac{E_{4}-1}{16} E_{1} E_{3} \equiv \frac{1}{2} \frac{E_{4}-1}{16} E_{3} \\
& \equiv \frac{E_{4}-1}{16} \frac{1}{2}\left(E_{3}-1\right) \equiv \frac{1}{2} \frac{E_{1}^{2}-1}{4} \frac{E_{4}-1}{16} \\
& \equiv \frac{1}{2} \frac{E_{6}-1}{8} \frac{E_{1}^{2}-1}{4} \equiv-\frac{1}{16} \frac{E_{1}^{2}-1}{4} .
\end{aligned}
$$

Remark 2.5. From the point of view of arithmetics, the product-type Kervaire elements $\left(x_{3}^{2}=\nu^{2}\right.$ and $\left.x_{7}^{2}=\sigma^{2}\right)$ are lucky exceptions: They happen to occur in the dimensions just low enough to be left unscathed by the congruence (ii) of Lemma 2.2.

\section{The 8-periodic families (iii) and (iv)}

According to $[\mathbf{1}]$, we have $e_{\mathbb{C}}\left(\mu_{8 k+1}\right)=1 / 2$, and this is the only possible non-trivial value of $e_{\mathbb{C}}$ in dimension $8 k+1$. Thus, we judiciously choose

$$
f\left(\mu_{8 k+1} \mu_{8 k^{\prime}+1}\right) \equiv \frac{1}{2} \frac{E_{1} E_{4}^{k}-1}{2} \in \underline{\underline{D}}_{4\left(k+k^{\prime}\right)+2}^{\Gamma} \otimes \mathbb{Q} / \mathbb{Z}
$$

but

$$
\frac{1}{2} \frac{E_{1} E_{4}^{k}-1}{2}-\frac{1}{2} \frac{E_{1}-1}{2}=\frac{E_{4}^{k}-1}{4} E_{1} \in \mathbb{Z} \llbracket q \rrbracket
$$

yields (iii). For (iv), we may assume that $e_{\mathbb{C}}\left(x_{8 k^{\prime}-1}\right) \equiv B_{4 k^{\prime}} / 8 k^{\prime} \bmod \mathbb{Z}$, hence

$$
f\left(\mu_{8 k+1} x_{8 k^{\prime}-1}\right) \equiv \frac{1}{2} \frac{B_{4 k^{\prime}}}{8 k^{\prime}}\left(E_{4 k^{\prime}}-1\right) \equiv \frac{1}{2} \sum \sigma_{4 k^{\prime}-1}(n) q^{n} \in \underline{\underline{D}}_{4\left(k+k^{\prime}\right)+1}^{\Gamma} \otimes \mathbb{Q} / \mathbb{Z},
$$

which is congruent to the desired result by virtue of Lemma 2.2 .

Remark 2.6. In order to express (iv) in terms of $E_{1}$ and $E_{3}$, it is useful to work out 
the following relation modulo $\underline{\underline{D}}_{4 k+1}^{\Gamma}$ :

$$
\begin{aligned}
\frac{1}{2} \frac{E_{4}-1}{16} & =\frac{1}{2}\left[\left(\frac{E_{1}^{2}-1}{4}\right)^{2}+\frac{1}{2} \frac{E_{1}^{2}-1}{4}+\frac{1}{2}\left(E_{1}^{4}-E_{1} E_{3}\right)\right] \\
& =\frac{1}{2}\left(\frac{E_{1}^{2}-1}{4}\right)^{2}+\frac{1}{2}\left[\frac{1}{2} \frac{E_{1}^{2}-1}{4}-\frac{1}{2}\left(E_{3}-1\right)\right]-\frac{1}{2} \frac{E_{1}-1}{2} E_{3}+\frac{E_{1}^{4}-1}{4} \\
& \equiv \frac{1}{2}\left(\frac{E_{1}^{2}-1}{4}\right)^{2}+\frac{1}{2}\left[\frac{1}{2} \frac{E_{1}^{2}-1}{4}-\frac{1}{2}\left(E_{3}-1\right)\right]+\frac{1}{2} \frac{E_{1}-1}{2} \frac{E_{1}^{2}-1}{4}
\end{aligned}
$$

furthermore, we observe that for $k \geqslant 2$ the first summand can be dropped due to $\frac{1}{2}\left(\frac{E_{1}^{2}-1}{4}\right)^{2} \equiv \frac{1}{2}\left(E_{3}-1\right)^{2} \equiv \frac{1}{2} E_{3}^{2} \equiv \frac{1}{2} E_{1}^{4 k-5} E_{3}^{2}$.

Remark 2.7. The elements occurring in part (iii) and (iv) of the theorem also allow detection via the invariants $d_{\mathbb{R}}$ and $e_{\mathbb{R}}$, respectively, cf. [1]. While for low values of $k$, $k^{\prime}$, the non-triviality of $f$ in these cases is easily checked by hand, the author does not know how to establish non-triviality for all values of $k, k^{\prime}$ in a direct manner.

\section{The generic situation}

For $(\mathrm{v})$, we may assume that $e_{\mathbb{C}}\left(x_{8 k+3}\right)$ is represented by $B_{4 k+2} /(4 k+2)$, so, for $k>0$, we have

$$
f\left(x_{8 k+3} \mu_{8 k^{\prime}+1}\right) \equiv \frac{1}{2} \frac{B_{4 k+2}\left(1-E_{4 k+2}\right)}{4 k+2}=\sum \sigma_{4 k+1}(n) q^{n},
$$

whereas for $k=0$ we have

$$
\begin{aligned}
f\left(x_{3} \mu_{8 k^{\prime}+1}\right) & \equiv \frac{1}{2} \frac{E_{1}^{2}-1}{12} \equiv \frac{1}{2}\left\{\frac{E_{1}^{2}-1}{12}+E_{4}^{k^{\prime}} E_{3}-1\right\} \\
& \equiv \frac{1}{2}\left\{\frac{E_{1}^{2}-1}{12}+E_{3}-1\right\} \bmod \underline{\underline{D}}_{4 k^{\prime}+3}^{\Gamma},
\end{aligned}
$$

which expands integrally by Lemma 2.3 .

Turning to (vi) and excluding the cases $k=k^{\prime}=1$ and $k=k^{\prime}=2$, we assume that $k \leqslant k^{\prime} ;$ thus

$$
f\left(x_{4 k-1} x_{4 k^{\prime}-1}\right) \equiv\left(\epsilon\left(k^{\prime}\right) \sum \sigma_{2 k^{\prime}-1}(n) q^{n}\right) \cdot\left(\epsilon(k) \frac{B_{2 k}}{4 k}\right),
$$

where $\epsilon(k)=1$ for $k$ even and two otherwise.

The theorem of von Staudt-Clausen [2] allows the computation of the denominator of the Bernoulli numbers. More precisely, let $j_{2 k}$ denote the denominator of $B_{2 k} / 2 k$; if $(p-1) p^{n-1} \mid 2 k$, then $p^{n} \mid j_{2 k}$, and this result is sharp. But by Lemma 2.2 (i) we have:

$$
p^{-n} \sum \sigma_{2 k^{\prime}-1+2 k}(r) q^{r} \equiv p^{-n} \sum \sigma_{2 k^{\prime}-1}(r) q^{r} \text { if }(p-1) p^{n-1} \mid 2 k,
$$

and the LHS is $p^{-n} G_{2 k+2 k^{\prime}}$ plus a constant, hence vanishes mod $\underline{\underline{D}}_{2\left(k+k^{\prime}\right)}$.

Similarly, if $k=2 l=\left(2 n^{\prime}+1\right) 2^{m+1}, 2^{m+4} \mid 2 j_{4 l}$ (and $2 j_{4 l}$ is precisely the order of 
$\operatorname{Im} J$ in dimension $8 l-1$ ), and part (ii) of Lemma 2.2 yields:

$$
\frac{1}{2^{4+m}}\left(\sum \sigma_{2 k^{\prime}-1+4 l}(r) q^{r}-\sum \sigma_{2 k^{\prime}-1}(r) q^{r}\right) \equiv 0 \quad \bmod \mathbb{Z} \llbracket q \rrbracket .
$$

This completes the proof.

\section{References}

[1] J.F. Adams, On the groups $J(X)$. IV, Topology 5 (1966), 21-71.

[2] T.M. Apostol, Introduction to Analytic Number Theory, Undergrad. Texts Math., Springer-Verlag, New York, 1976.

[3] M.F. Atiyah, V.K. Patodi, and I.M. Singer, Spectral asymmetry and Riemannian geometry. I, Math. Proc. Cambridge Philos. Soc. 77 (1975), 43-69.

[4] M.F. Atiyah, V.K. Patodi, and I.M. Singer, Spectral asymmetry and Riemannian geometry. II, Math. Proc. Cambridge Philos. Soc. 78 (1975), no. 3, 405432.

[5] U. Bunke and N. Naumann, The f-invariant and index theory, Manuscripta Math. 132 (2010) 365-397.

[6] P.E. Conner and E.E. Floyd, The Relation of Cobordism to K-Theories, Lect. Notes Math., No. 28, Springer-Verlag, Berlin, 1966.

[7] Ch. Deninger and W. Singhof, The e-invariant and the spectrum of the Laplacian for compact nilmanifolds covered by Heisenberg groups, Invent. Math. $\mathbf{7 8}$ (1984), no. 1, 101-112.

[8] J. Hornbostel and N. Naumann, Beta-elements and divided congruences, Amer. J. Math. 129 (2007), no. 5, 1377-1402.

[9] G. Laures, The topological q-expansion principle, Topology 38 (1999), no. 2, $387-425$.

[10] G. Laures, On cobordism of manifolds with corners, Trans. Amer. Math. Soc. 352 (2000), no. 12, 5667-5688 (electronic).

[11] H.R. Miller, D.C. Ravenel, and W.S. Wilson, Periodic phenomena in the Adams-Novikov spectral sequence, Ann. of Math. (2) 106 (1977), no. 3, 469516.

[12] D.C. Ravenel, Complex Cobordism and Stable Homotopy Groups of Spheres. 2nd ed., AMS Chelsea Publishing, Providence, RI, 2004.

[13] H. von Bodecker, On the geometry of the f-invariant, Ph.D. thesis, RuhrUniversität Bochum, 2008.

Hanno von Bodecker hvb@math.uni-bielefeld.de

Fakultät für Mathematik, Universität Bielefeld, Postfach 100131, D-33501 Bielefeld, Germany 